\title{
Early high-sodium solid diet does not affect sodium intake, sodium preference, blood volume and blood pressure in adult Wistar-Kyoto rats
}

\author{
Marcin Ufnal*, Adrian Drapala, Mariusz Sikora and Tymoteusz Zera \\ Department of Experimental and Clinical Physiology, The Medical University of Warsaw, 26/28 Krakowskie Przedmiescie \\ Street, Warsaw, 00-927, Poland
}

(Received 18 October 2010 - Revised 17 December 2010 - Accepted 20 December 2010 - First published online 17 May 2011)

\begin{abstract}
A high-Na diet may lead to the development of hypertension in both humans and rats; however, the causes of Na intake in amounts greater than physiologically needed as well as the mechanisms whereby high-Na food elevates blood pressure are not clear. Therefore, we decided to test the hypothesis that a high-Na diet introduced after suckling affects $\mathrm{Na}$ intake, food preference, resting blood pressure and blood volume in adult rats. Male Wistar-Kyoto (WKY) rats, 4 weeks old, were divided into three groups and placed on either a high-Na (3.28\%), a medium-Na $(0.82 \%)$ or a regular diet $(0.22 \%)$ with the same energy content for 8 weeks. Subsequently, food preference, resting arterial blood pressure, blood volume, plasma osmolality and Na blood level were evaluated. When offered a choice of diets, all the groups preferred the regular chow, and there was no significant difference in total $\mathrm{Na}$ intake between the groups. When the rats experienced the change from their initial chow to a new one with different Na content, they continued to eat the same amount of food. Body weight, resting arterial blood pressure, blood volume, plasma osmolality and $\mathrm{Na}$ blood level were comparable between the groups. In conclusion, the results show that a high-Na diet introduced immediately after suckling does not affect Na preference and Na intake in adult WKY rats. Furthermore, the findings provide evidence that both blood volume and arterial blood pressure are highly protected in normotensive rats on a high-Na diet.
\end{abstract}

Key words: High-sodium diet: Food preference: Blood volume: Hypertension

Both humans and rats demonstrate an innate appetite for salt and ingest it in excess of their apparent needs. A high-Na diet may result in hypertension; however, there are significant individual differences between humans as well as between strains of rats in sensitivity to salt ${ }^{(1,2)}$. The causes of $\mathrm{Na}$ intake in amounts greater than physiologically needed as well as the mechanisms whereby salt intake elevates blood pressure are not clear. Several causative links between a high-Na diet and the development of hypertension have been postulated. For example, it has been suggested that a high-Na diet increases the concentration of $\mathrm{Na}$ in the plasma or the cerebrospinal fluid. These in turn may stimulate peripheral or brain mechanisms leading to the expansion of blood volume, increased cardiac output or peripheral resistance, all of which may elevate arterial blood pressure ${ }^{(3,4)}$. Interestingly, there is strong evidence for the involvement of common pathways in the regulation of $\mathrm{Na}$ intake and arterial blood pressure. It has been found that aldosterone and angiotensin II, two hormones that play a pivotal role in the regulation of blood pressure, have also a significant influence on $\mathrm{Na}$ intake ${ }^{(5)}$.
Total intake of $\mathrm{Na}$ depends on $\mathrm{Na}$ appetite, also referred to as $\mathrm{Na}$ hunger, and on $\mathrm{Na}$ preference. The latter can be described as a relative measure of the ingestion of high-Na food in comparison with the ingestion of low-Na food. An increased preference for a high-Na diet usually signifies the increased intake of $\mathrm{Na}$, on condition that total food intake is not affected. Pioneer research studies on $\mathrm{Na}$ appetite were undertaken by Richter ${ }^{(6)}$, who found that adrenalectomised rats significantly increase their intake of saline solutions in preference to water. Later, Epstein \& Stellar ${ }^{(7)}$ demonstrated that salt appetite is innate and does not depend on learning. Several lines of evidence suggest that the development of salt taste and $\mathrm{Na}$ preference may be modified during early stages of life by changes in dietary $\mathrm{Na}$ content. However, no specific pattern for $\mathrm{Na}$ preference variation has been established, since both excess and restricted early Na dietary availability have been found to reduce or increase, or do not change $\mathrm{Na}$ intake in adult rats ${ }^{(8)}$.

In previous studies, authors have tested $\mathrm{Na}$ preference and appetite by presenting rats with $\mathrm{NaCl}$ solutions. In the present

Abbreviations: WKY, Wistar-Kyoto; group H, high-Na group; group L, low-Na group; group M, medium-Na group; group N, normal-Na group.

*Corresponding author: Dr M. Ufnal, fax +48 2282645 86, email marcinufnal@poczta.onet.pl 
study, we decided to determine Na preference by exposing rats to solid food. This approach is important since under most conditions of life, Na intake depends on the ingestion of solid food rather than on drinking salty liquids. In contrast to the latter, the consumption of salty food is associated with the ingestion of a number of nutrients and intake of energy. Therefore, it seems likely that $\mathrm{Na}$ preference as well as cardiovascular responses to the ingestion of a high $\mathrm{Na}$ load may depend not only on the size of the load but also on the ingestion of other compounds.

Recently, excessive $\mathrm{Na}$ intake has been reappraised as the major risk factor of hypertension; however, a number of studies have highlighted the importance of other factors such as genetic predispositions, intake of other ions and acquired disturbances in the mechanisms of handling $\mathrm{Na}^{(1)}$. In order to evaluate the role of early high-Na diet as a single factor that may affect $\mathrm{Na}$ intake, blood pressure and blood volume, we performed the experiments on healthy, young, normotensive Wistar-Kyoto (WKY) rats.

\section{Experimental methods}

The experimental design was approved by the Ethical Committee of the Medical University of Warsaw.

\section{Animals}

After suckling, 28-d-old male WKY rats (Animal Breeding Department, the Medical University of Warsaw) were assigned to three dietary regimens with the same energy content $(12 \mathrm{MJ} / \mathrm{kg}$ ) but with different $\mathrm{Na}$ content. Specifically, the rats were placed either on a high-Na $(3 \cdot 28 \%$, group $\mathrm{H})$, a medium-Na $(0.82 \%$, group $\mathrm{M})$ or a normal-Na $(0.22 \%$, groups $\mathrm{N}$ and $\mathrm{N}^{\prime}$ ) pellet chow (Labofeed, Kcynia, Poland). The rats were maintained with free access to a specific diet and tap water ad libitum for 8 weeks. Subsequently, the animals were assigned to either a choice preference test or a sequence preference test. After the tests, all the rats returned to their living cages and were maintained on their initial diet for another week. Afterwards, resting arterial blood pressure, blood volume, plasma osmolality, blood $\mathrm{K}$ and $\mathrm{Na}$ levels were measured.

\section{Preference tests}

Choice test. In the choice test, each rat was put into a separate metabolism cage with free access to tap water and its initial chow which was presented in two identical feeding boxes. The first day was allowed for adaptation to the new environment. The next day, the chow from one of the boxes was changed, so that the animals from groups $\mathrm{N}(n 7)$ and $\mathrm{H}$ ( $n$ 7) had a choice between the high-Na diet and the normal-Na diet, whereas the rats from groups $\mathrm{N}^{\prime}(n 7)$ and $\mathrm{M}$ ( $n$ 7) were presented with the medium-Na and the normal-Na chow for the following $2 \mathrm{~d}$. The position of the feeding boxes was changed every $24 \mathrm{~h}$ in order to minimise position-related preference.
Sequence test. In the sequence test, the rats were put individually into metabolism cages with free access to tap water and their initial diet. The first day was allowed for adaptation to the new environment. During two initial days, the rats continued to eat their initial diet. The next day, the chow was changed so that groups $\mathrm{H}(n 7)$ and $\mathrm{M}(n 7)$ were offered the normal-Na diet, group $\mathrm{N}(n 7)$ received the high-Na chow, while group $\mathrm{N}^{\prime}(n 7)$ was put on the medium-Na chow.

\section{Arterial blood pressure, blood volume and electrolyte measurements}

Resting mean arterial blood pressure was recorded for $30 \mathrm{~min}$, on freely moving rats in their living cages, during the first $3 \mathrm{~h}$ of the light period. The detailed methodology has been described elsewhere ${ }^{(9)}$. Blood volume was calculated according to the following formula: volume $(\mathrm{ml})=$ (plasma volume) $(100-$ percentage of blood cells $) \times 100$. Measurements of plasma volume were performed according to the classical method of determining the distribution of Evans blue; the detailed methodology has been described elsewhere ${ }^{(10)}$. In short, blood was withdrawn from the femoral vein immediately before and 10 and $30 \mathrm{~min}$ after the injection of the dye into the other femoral vein. The Evans blue plasma concentrations were measured by photometry at the wavelength of $610 \mathrm{~nm}$ against blank plasma. Blood samples for the measurements of plasma osmolality, blood $\mathrm{K}$ and $\mathrm{Na}$ were obtained from the tip of the tail during the first hour of the light period.

\section{Statistics}

Results are expressed as means with their standard errors. In the preference tests, measurements from two consecutive days were averaged, and the mean values were taken for analysis. The differences between the groups were analysed using ANOVA, followed by Tukey's test (Statistica software, version 9; StatSoft Polska Sp. z O.o., Krakow, Poland). Values of $P<0.05$ were considered to be significant.

\section{Results}

After 8 weeks of maintaining the rats on their initial diets, there were no significant differences between the groups in body weight (group N 288.3 (sE 9.0) g; group $\mathrm{N}^{\prime} 291 \cdot 0$ (SE 9.8) g; group M 293.6 (SE 10.0) g; group H 291.9 (SE 10.4) g) and food intake (group N 8.24 (SE 0.41) g/100g body weight, group $\mathrm{N}^{\prime} 8.45(\mathrm{SE} 0.35) \mathrm{g} / 100 \mathrm{~g}$ body weight, group M 8.47 (SE 0.61) g/100 g body weight, group H 8.65 (sE 0.50) g/100 g body weight).

\section{Choice test}

Total sodium intake. There was no significant difference in total $\mathrm{Na}$ intake between the groups (group N 0.032 (sE 0.0019) g/100 g body weight, group $\mathrm{N}^{\prime}$ 0.030 (SE 0.0016) $\mathrm{g} / 100 \mathrm{~g}$ body weight, group M 0.028 (sE 0.0014) g/100 g body weight, H 0.031 (SE 0.0042) g/100 g body weight). 


\section{Food preference}

Group $N$ v. group $H$. Factorial ANOVA (groups $\mathrm{N}$ and $\mathrm{H} \times$ normal-Na and high-Na chow) revealed a significant difference in food intake within the groups $(F(1,24)$ 402.4, $P<0 \cdot 001)$. Both groups ingested significantly greater amount of the normal-Na chow than the high-Na chow (group $\mathrm{N}$, $P<0 \cdot 001$; group H, $P<0 \cdot 0001$; by Tukey's test, Fig. 1(a)).

Group $N^{\prime}$ v. group $M$. Factorial ANOVA (groups $N^{\prime}$ and $\mathrm{M} \times$ normal-Na and medium-Na chow) revealed a significant difference in food intake within the groups ( $F(1,24)$ 215.9, $P<0.001$ ). Both groups, $\mathrm{N}^{\prime}$ and $\mathrm{M}$, ingested significantly greater amount of the normal-Na than the medium-Na chow (group $\mathrm{N}^{\prime}, P<0.001$; group $\mathrm{M}, P<0.001$; by Tukey's test, Fig. 1(b)).

Sequence test. There was no significant difference between group $\mathrm{N}$ and group $\mathrm{H}$ in the ingestion of either the normal-Na chow or the high-Na chow (Fig. 1(c)). Similarly, there was no difference between group $\mathrm{N}^{\prime}$ and group $\mathrm{M}$ in the ingestion of either the normal-Na or the medium-Na chow (Fig. 1(d)).

\section{Arterial blood pressure, blood volume and electrolyte measurements}

There were no significant differences between the groups in the following parameters: resting mean arterial blood pressure, heart rate, blood volume, plasma volume, haematocrit, plasma osmolality, blood $\mathrm{Na}$ and $\mathrm{K}$ levels (Table 1 ).

\section{Discussion}

The results show that an early high-Na diet fails to affect $\mathrm{Na}$ intake, $\mathrm{Na}$ preference, blood volume and resting arterial blood pressure in WKY rats. These findings imply that $\mathrm{Na}$ intake in adult rats depends more on an innate $\mathrm{Na}$ appetite than on the experience of the rats with high-Na food during early stages of life. Furthermore, the study provides evidence that both blood volume and arterial blood pressure are closely regulated in normotensive rats that ingest high $\mathrm{Na}$ loads.

In the choice test, all groups preferred the regular diet and showed aversion to food with a higher Na content. Moreover, there was no significant difference in total $\mathrm{Na}$ intake between (a)

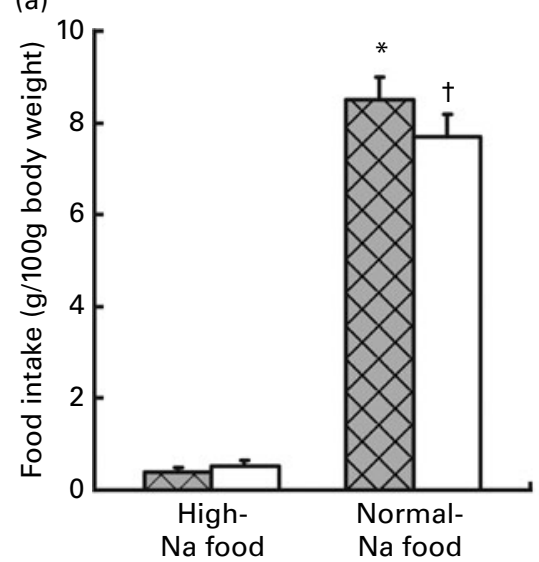

(c)

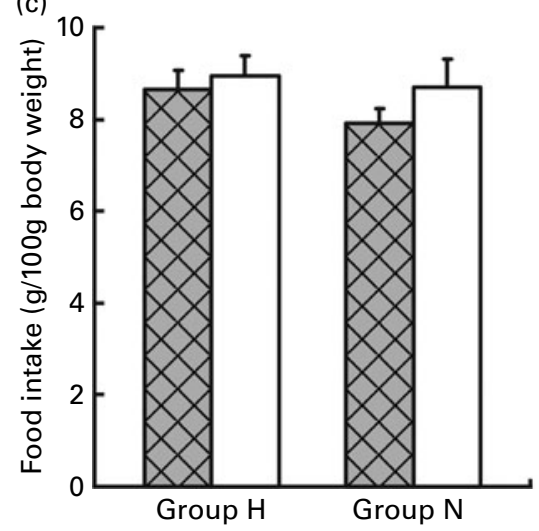

(b)

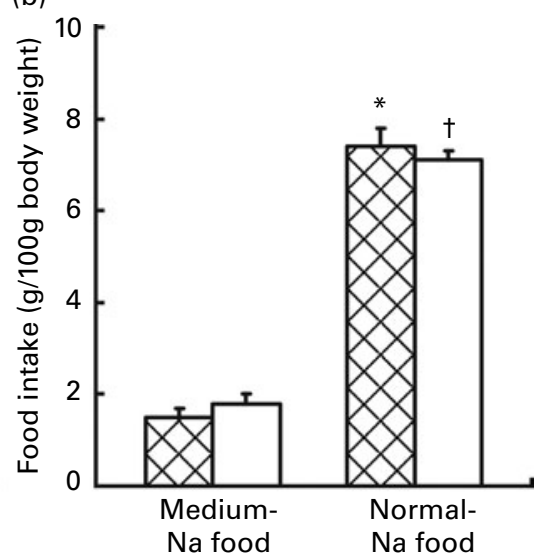

(d)

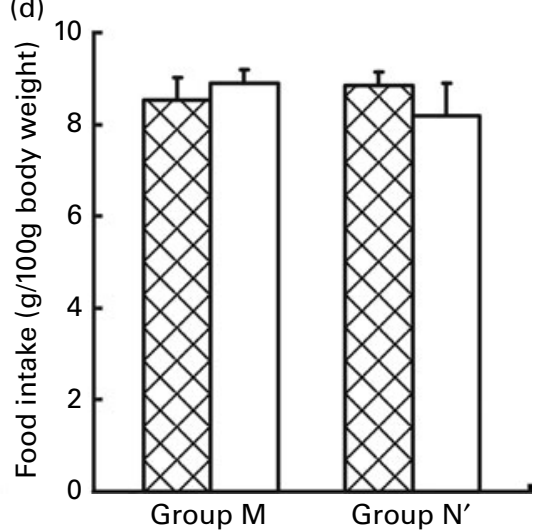

Fig. 1. Ingestion of food in the preference tests. Values are means, with standard errors represented by vertical bars. Choice test: (a) ingestion of food ( $\mathrm{g} / 100 \mathrm{~g}$ body weight) by rats maintained previously on a normal-Na diet (group $\mathrm{N}, \square$ ) $v$. rats maintained previously on a high-Na diet (group $\mathrm{H}$, $\otimes$ ). ${ }^{*}$ Mean values were significantly different for ingestion of normal-Na food $v$. high-Na food in group $\mathrm{H}(P<0.001)$. † Mean values were significantly different for ingestion of normal-Na food $v$. high-Na food in group $\mathrm{N}(P<0.001)$. (b) Ingestion of food ( $\mathrm{g} / 100 \mathrm{~g}$ body weight) by rats maintained previously on a normal-Na diet (group $\left.\mathrm{N}^{\prime}, \square\right) v$. rats maintained previously on a medium-Na diet (group $\mathrm{M}, \otimes)$. * Mean values were significantly different for ingestion of normal-Na food $v$. medium-Na food in group $\mathrm{M}(P<0.001)$. † Mean values were significantly different for ingestion of normal-Na food $v$. medium-Na food in group $\mathrm{N}(P<0.001)$. Sequence test: (c) ingestion of food ( $\mathrm{g} / 100 \mathrm{~g}$ body weight) by rats maintained previously on a normal-Na diet, $\square$ (group $\mathrm{N}$ ) $v$. rats maintained previously on a high-Na diet, $\otimes$ (group $\mathrm{H}$ ). (d) Ingestion of food ( $\mathrm{g} / 100 \mathrm{~g}$ body weight) by rats maintained previously on a normal-Na diet, $\square$ (group $\mathrm{N}^{\prime}$ ) $v$. rats maintained previously on a medium-Na diet, $\otimes($ group $\mathrm{M})$. 
Table 1. Measurements of arterial blood pressure, blood volume and blood electrolytes in rats maintained on a normal-sodium diet (group N), a medium-sodium diet (group M) and a high-sodium diet (group H) (Mean values with their standard errors)

\begin{tabular}{|c|c|c|c|c|c|c|}
\hline & \multicolumn{2}{|c|}{ Group N } & \multicolumn{2}{|c|}{ Group M } & \multicolumn{2}{|c|}{ Group H } \\
\hline & Mean & SE & Mean & SE & Mean & SE \\
\hline Arterial blood pressure $(\mathrm{mmHg})$ & 115 & $2 \cdot 6$ & 115 & 3.4 & 116 & $3 \cdot 1$ \\
\hline Heart rate (beats/min) & 345 & 11 & 332 & 13 & 340 & 18 \\
\hline Blood volume (ml/100 g body wt) & 8.8 & 0.23 & 8.9 & 0.19 & 8.5 & 0.25 \\
\hline Plasma volume (ml/100 $\mathrm{g}$ body $\mathrm{wt})$ & $5 \cdot 0$ & 0.14 & $5 \cdot 2$ & 0.11 & $5 \cdot 1$ & 0.14 \\
\hline Haematocrit ( $\%$ of blood cells) & $42 \cdot 4$ & $1 \cdot 2$ & 40.9 & 0.8 & $39 \cdot 7$ & 0.9 \\
\hline Plasma osmolality $(\mathrm{mOsm} / \mathrm{kg})$ & 292 & $6 \cdot 1$ & 291 & 3.0 & 292 & 3.7 \\
\hline Blood Na level $(\mathrm{mmol} / \mathrm{l})$ & 139 & $1 \cdot 1$ & 139 & 0.8 & 141 & 0.9 \\
\hline Blood K level (mmol/l) & 4.5 & 0.36 & 4.5 & 0.21 & 4.9 & 0.15 \\
\hline
\end{tabular}

the groups. Therefore, we conclude that in contrast to $\mathrm{Na}$ depletion which increases $\mathrm{Na}$ intake ${ }^{(11,12)}$, the ingestion of high-Na food in early life fails to affect $\mathrm{Na}$ intake in adult rats. The present results are somehow different from those obtained from the evaluation of $\mathrm{Na}$ preference by exposure of rats to saline solutions. Namely, Contreras \& Kosten ${ }^{(13)}$ found that rat offspring raised on a high-Na diet exhibit enhanced salt ( $\mathrm{NaCl}$ solutions) preference when adult. In addition, Priehs et al. ${ }^{(14)}$ showed that rats maintained on a high-Na diet increase their intake of $\mathrm{Na}$ when given a low$\mathrm{Na}$ solution. However, similar to the present results, they reported that rats showed lower preference for a high-Na diet ( $\mathrm{NaCl}$ solutions). Therefore, it appears that the way in which $\mathrm{Na}$ is offered may have an impact on the outcome of the tests evaluating $\mathrm{Na}$ intake and $\mathrm{Na}$ preference. It has been found that the gustatory sense plays a key role in the control of salt intake ${ }^{(15)}$. Furthermore, several lines of evidence suggest that salt taste depends on an interaction between $\mathrm{Na}$ ions and amiloride-sensitive $\mathrm{Na}$ channels as well as a vanilloid receptor-1 variant ${ }^{(16,17)}$. It is possible that there is a difference between the patterns of stimulation of the receptors by $\mathrm{Na}$ ions contained in $\mathrm{NaCl}$ solutions and $\mathrm{Na}$ from solid food. In contrast to saline, solid food contains a number of compounds that may interact with either $\mathrm{Na}$ ions or the receptors.

Furthermore, the results suggest that the primary imperative for rats that experience a change of $\mathrm{Na}$ content in their diet is to continue to meet their requirements for energy or vital nutrients rather than to maintain the intake of $\mathrm{Na}$ at the same level. Therefore, we hypothesise that in normotensive rats, 'a $\mathrm{Na}$ intake set point' is not affected by a high-Na diet introduced immediately after suckling and that it is secondary to 'an energy set point' or requirements for vital nutrients. This is based on the following premises. First, after 8 weeks of maintaining the rats on their initial diets with the same energy but with different $\mathrm{Na}$ content, there were no significant differences between the groups in total food ingestion and body weight. Second, in the sequence test, the rats which experienced the change from their initial diet to a new one with different $\mathrm{Na}$ content continued to eat the same amount of food and thus significantly changed their $\mathrm{Na}$ intake. The hypothesis, however, cannot be extrapolated to Na-deficit conditions and requires further testing using low-Na and high-Na diets with different energy levels.

High $\mathrm{Na}$ intake is widely considered the major risk factor of hypertension; however, the causative mechanisms are still not clear. It has been postulated that an increased $\mathrm{Na}$ ingestion may elevate $\mathrm{Na}$ blood level, and that even a slight increase in $\mathrm{Na}$ blood level, by $1-3 \mathrm{mmol} / \mathrm{l}$, may result in hypertension due to the expansion of the extracellular fluid and blood volume $^{(3)}$. In our experiments, neither the medium- nor the high-Na diet affected the resting arterial blood pressure or the $\mathrm{Na}$ blood level. The results are consistent with our previous findings ${ }^{(18)}$ and those of other investigators ${ }^{(2,19)}$ who showed that a high-Na diet does not affect arterial blood pressure in WKY rats.

Additionally, the present study provides new evidence that a high-Na diet does not affect plasma and blood volumes in WKY rats. Although there are several lines of evidence that a high-Na diet affects the structure and the regulation of the circulatory system in WKY rats ${ }^{(2,18,19)}$ as well as promotes the development of hypertension in NaCl-sensitive spontaneously hypertensive rats (which are derived from WKY rats $)^{(19)}$, the present study shows that in normotensive rats, both arterial blood pressure and blood volume are highly protected.

The limitation of $\mathrm{Na}$ content in food has historically been the core of nutritional polices. However, epidemiological data and numerous interventional studies in humans and animals provide evidence that the development of hypertension due to high salt intake depends on genetic make-up, acquired impairment in the mechanisms of handling $\mathrm{Na}$ and the intake of other ions ${ }^{(1,20,21)}$. The present study supports the notion that a high $\mathrm{Na}$ intake, as a single factor, is insufficient for the development of hypertension in young healthy animals.

In conclusion, the present study reveals that a high-Na diet introduced immediately after suckling does not affect $\mathrm{Na}$ preference and $\mathrm{Na}$ intake in adult WKY rats. The findings also indicate that both blood volume and arterial blood pressure are closely regulated in normotensive rats that ingest high Na loads. 


\section{Acknowledgements}

The present study was supported by the Medical University of Warsaw. The authors have no conflict of interest to disclose. M. U. and T. Z. designed the research, conducted the study and wrote the manuscript. A. D. and M. S. analysed the data and wrote the manuscript. M. U. had the primary responsibility for the final content. All authors read and approved the final manuscript.

\section{References}

1. McCarron DA (1998) Diet and blood pressure - the paradigm shift. Sci Tech Froid 281, 933-934.

2. Leenen FH \& Yuan B (1998) Dietary-sodium-induced cardiac remodeling in spontaneously hypertensive rat versus Wistar-Kyoto rat. J Hypertens 16, 885-892.

3. de Wardener HE, He FJ \& MacGregor GA (2004) Plasma sodium and hypertension. Kidney Int 66, 2454-2466.

4. Huang BS, Van Vliet BN \& Leenen FH (2004) Increases in $\mathrm{CSF}\left[\mathrm{Na}^{+}\right]$precede the increases in blood pressure in Dahl $\mathrm{S}$ rats and SHR on a high-salt diet. Am J Physiol Heart Circ Physiol 287, H1160-H1166.

5. Stellar E (1993) Salt appetite: its neuroendocrine basis. Acta Neurobiol Exp 53, 475-484.

6. Richter CP (1936) Increased salt appetite in adrenalectomized rats. Am J Physiol 115, 155-161.

7. Epstein AN \& Stellar E (1955) The control of salt preference in the adrenalectomized rat. I Comp Physiol Psychol 48, $167-172$

8. Lesham M (1999) The ontogeny of salt hunger in the rat. Neurosci Biobehav Rev 23, 649-659.

9. Ufnal M, Dudek M \& Szczepanska-Sadowska E (2006) Inhibition of brain nitric oxide synthesis enhances and prolongs the hypertensive effect of centrally administered interleukin-1beta in rats. Cytokine 7, 166-170.
10. Gibson JG \& Evans WA (1937) Clinical studies of the blood volume I. Clinical application of a method employing the azo dye "Evans blue" and spectrophotometer. J Clin Invest 16, 301-316.

11. Sakai RR, Frankmann SP, Fine WB, et al. (1989) Prior episodes of sodium depletion increase the need-free sodium intake of the rat. Behav Neurosci 103, 186-192.

12. Thaw AK, Frankmann S \& Hill DL (2000) Behavioral taste responses of developmentally NaCl-restricted rats to various concentrations of NaCl. Behav Neurosci 144, 437-441.

13. Contreras RJ \& Kosten T (1983) Prenatal and early postnatal $\mathrm{NaCl}$ intake modifies the solution preferences of adult rats. J Nutr 113, 1051-1062.

14. Priehs TW, Mooney KJ \& Bernard RA (1991) High dietary sodium enhances gustatory nerve activity and behavioral responses to $\mathrm{NaCl}$. Am J Physiol 30, R52-R58.

15. Nachman M \& Valentino DA (1966) Roles of taste and postingestional factors in the satiation of sodium appetite in rats. J Comp Physiol Psychol 62, 280-283.

16. Heck GL, Mierson S \& DeSimone JA (1984) Salt taste transduction occurs through an amiloride-sensitive sodium transport pathway. Science 223, 403-405.

17. Lyall V, Heck GL, Vinnikova AK, et al. (2004) The mammalian amiloride-insensitive non-specific salt taste receptor is a vanilloid receptor-1 variant. $J$ Physiol 558, 147-159.

18. Zera T \& Ufnal M (2005) Enhanced pressor response to centrally administered vasopressin in WKY rats on high sodium diet. Acta Neurobiol Exp 65, 145-150.

19. Calhoun DA, Wyss JM \& Oparil S (1991) High $\mathrm{NaCl}$ diet enhances arterial baroreceptor reflex in NaCl-sensitive spontaneously hypertensive rats. Hypertension 17, 363-368.

20. Woolfson RG \& de Wardener HE (1996) Primary renal abnormalities in hereditary hypertension. Kidney Int $\mathbf{5 0}$, 717-731.

21. Hermansen K (2000) Diet, blood pressure and hypertension. Br J Nutr 83, Suppl. 1, S113-S119. 